\title{
Spectrometric and imaging measurements of a spectacular gravity wave event observed during the ALOHA-93 campaign
}

\author{
M.J. Taylor \\ Space Dynamics Laboratory and Physics Department, Utah State University \\ D.N. Turnbull and R.P. Lowe \\ Institute for Space and Terrestrial Science and Physics Department, The University of Western Ontario
}

\begin{abstract}
During the ALOHA-93 campaign coincident imaging and interferometric measurements of the near infrared and visible wavelength nightglow emissions were made from Haleakala Crater, Maui. On 10 October, 1993 a most unusual wave event was observed. This disturbance appeared as a sharp "front" followed by several conspicuous wave crests which progressed rapidly through the imager's field of view $\left(180^{\circ}\right)$. As the front passed overhead the interferometer detected a sudden jump in both the $\mathrm{OH}$ intensity (>50\%) and its rotational temperature $(20 \mathrm{~K})$ with the temperature increase leading the intensity by almost $15 \mathrm{~min}$. At the same time the imager registered a sharp decrease in the $\mathrm{OI}(557.7 \mathrm{~nm})$ emission intensity. A description of this remarkable event follows.
\end{abstract}

\section{Introduction}

Observations of gravity waves in the near infrared (NIR) and visible wavelength nightglow emissions (altitude $\mathbf{- 8 0 - 1 0 0 ~} \mathrm{km}$ ) have been reported by a number of researchers over the past two decades. The measurements have primarily been made using instruments such as photometers and spectrometers (aimed at the zenith) [eg. Takahashi et al., 1985; Lowe et al., 1991] and by narrow field imagers (usually aimed at low elevations to enhance the line of sight signal) [eg. Armstrong, 1982; Taylor et al., 1987]. Both techniques have revealed independently a wealth of short period ( $<1$ hour) wave structure in the mid- and low latitude mesosphere and lower thermosphere. However, coincident imaging and spectrometric observations of a common emission volume are relatively rare [Taylor et al., 1991]. Such measurements are highly complementary and permit the investigation of gravity wave events in considerable detail. In particular the relationship between the temperature and intensity perturbations induced by the passage of these waves is of considerable interest. During the ALOHA-93 campaign a suite of instruments was operated from Haleakala Crater, Maui, to investigate the dynamics of short period gravity waves in unprecedented detail. These included the University of Western Ontario infrared Michelson Interferometer (UWOMI-3) and the multi-wavelength, all-sky CCD imager of Utah State University. Coincident data were obtained on several nights and a variety of wave displays were observed. In this paper we present measurements of the transit of one spectacular event as recorded by these instruments and discuss its remarkable properties.

Copyright 1995 by the American Geophysical Union.

Paper number 95GL02948

0094-8534/95/95GL-02948\$03.00

\section{Observations}

During the period 6-23 October, 1993, joint imaging and spectrometric observations were made from the DOE Safeguard Facility, Haleakala Crater, Maui $\left(20.8^{\circ} \mathrm{N}, 156.2^{\circ} \mathrm{W}, 2970 \mathrm{~m}\right)$. Brief descriptions of these two instruments and their operational details are given in Taylor et al., [1995] and Lowe and Turnbull [1995]. The spectrometer (field of view $10^{\circ}$ ) measured the zenith intensity of several $\mathrm{OH}$ Meinel bands in the wavelength range $1000-1650 \mathrm{~nm}$, using a scan time of $23 \mathrm{~s}$. The all-sky imager recorded structure sequentially in the NIR OH(715-930 $\mathrm{nm})$ bands, the $\mathrm{OI}(557.7 \mathrm{~nm})$ and $\mathrm{Na}(589.2 \mathrm{~nm})$ lines and the $\mathrm{O}_{2}(0,1)$ Atmospheric band. The NIR OH filter incorporated a notch at $\sim 865 \mathrm{~nm}$ to limit contributions from the $\mathrm{O}_{2}(0,1)$ band. Measurements were made using a repetition rate of 3 and 5 min for the OI emission and $\sim 9$ min for the other emissions.

\section{October Wave Event}

The night of 10 October was characterized initially by a faint, but persistent, short horizontal wavelength $(\sim 15 \mathrm{~km})$ gravity wave propagating towards the $\mathrm{NE}$ in the $\mathrm{OI}$ emission layer (altitude $\sim 96 \mathrm{~km}$ ). Occasional small-scale, short-lived "ripple" waves were also observed in isolation at $\mathrm{OH}, \mathrm{Na}$ and $\mathrm{OI}$ wavelengths. However, around 09:30 UT an unusual disturbance was detected in the northwestern sky near the limit of the camera's field of view $(\sim 450 \mathrm{~km})$ in all four emissions. This disturbance appeared bright in the $\mathrm{OH}$ and $\mathrm{Na}$ emissions but dark in the $\mathrm{OI}$ and $\mathrm{O}_{2}$ emissions. By 10:30 UT the disturbance was resolved clearly as a series of elongated wave crests characterized by a leading sharp "front" propagating towards the SE. During the next hour the front moved rapidly over the Hawaiian Islands passing over the optical site and through the interferometer's field of view at $\sim 10: 50$ UT. Sequential images showed similar wave structure in each emission indicating that the disturbance extended throughout the upper mesosphere and lower thermosphere $(\sim 80-100 \mathrm{~km})$ region. Figure 1 shows this event as imaged in the NIR OH and the $\mathrm{OI}(557.7 \mathrm{~nm})$ nightglow emissions (the first two images are separated by $18 \mathrm{~min}$, while the second and third are separated by $9 \mathrm{~min}$ ). The data illustrate the spatial extent and temporal coherence of the wave packet and its rapid motion across the sky. By 11:30 UT the disturbance had progressed well to the SE of Maui and only faint evidence of its passage remained in the overhead sky.

At $\mathrm{OH}$ wavelengths this event appeared as a series of bright wave crests propagating through a "dark" almost structureless sky. However, in the OI emission it was manifested as a wave propagating into a "bright" nightglow region leaving an apparently depleted emission in its wake. The net effect was quite extraordinary with a marked reversal in contrast between the wave patterns as recorded at $\mathrm{OH}$ and $\mathrm{OI}$ wavelengths. Figure 1 

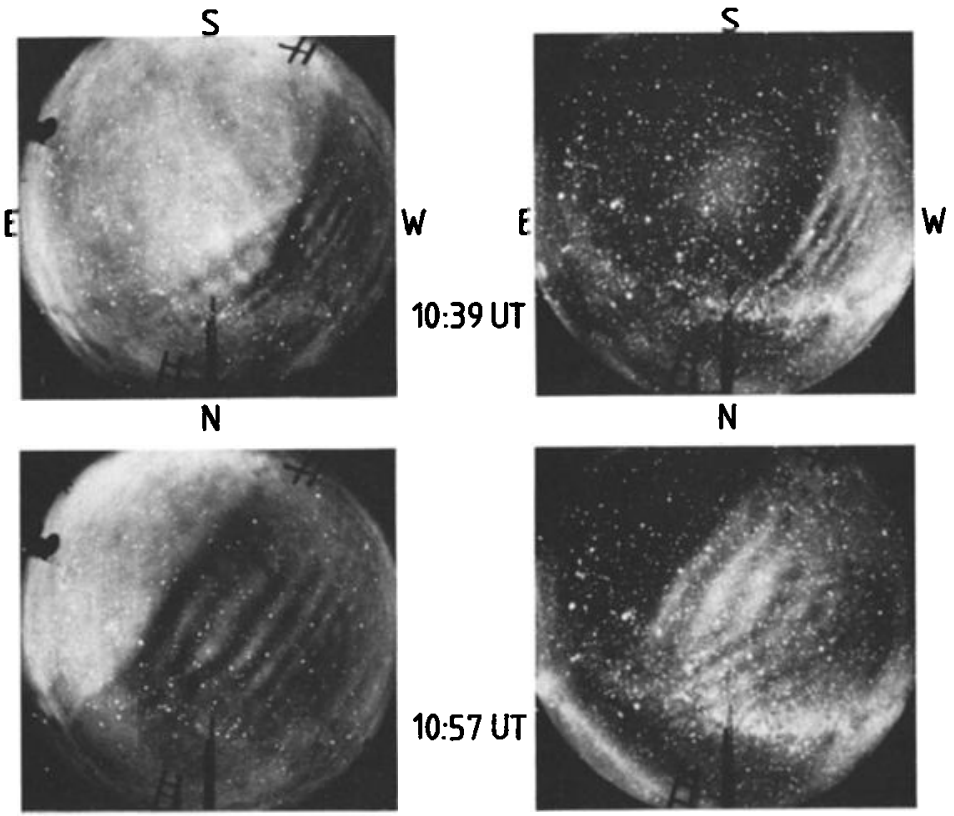

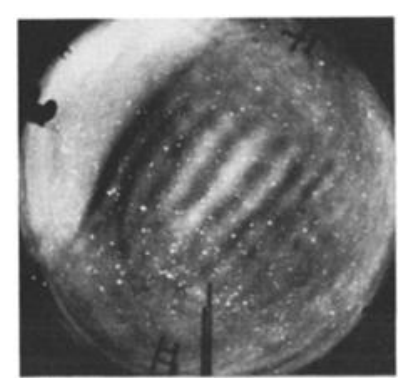

$0 I(557.7 \mathrm{~nm})$

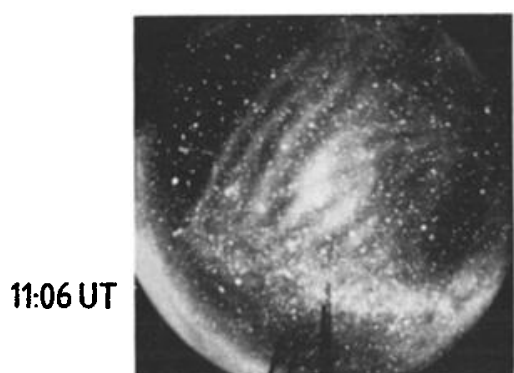

NIR OH

Figure 1. Sequence of all-sky CCD images showing the rapid transit of the wave event at NIR OH and $\mathrm{OI}(557.7 \mathrm{~nm}$ ) wavelengths (integration time for $\mathrm{OH}=20 \mathrm{~s}$ and $\mathrm{OI}=90 \mathrm{~s}$ ). Note the reversal in contrast of the wave pattern and the presence of the second, fainter wave motion $\left(\lambda_{h}=15 \mathrm{~km}\right)$ in the Ol images.

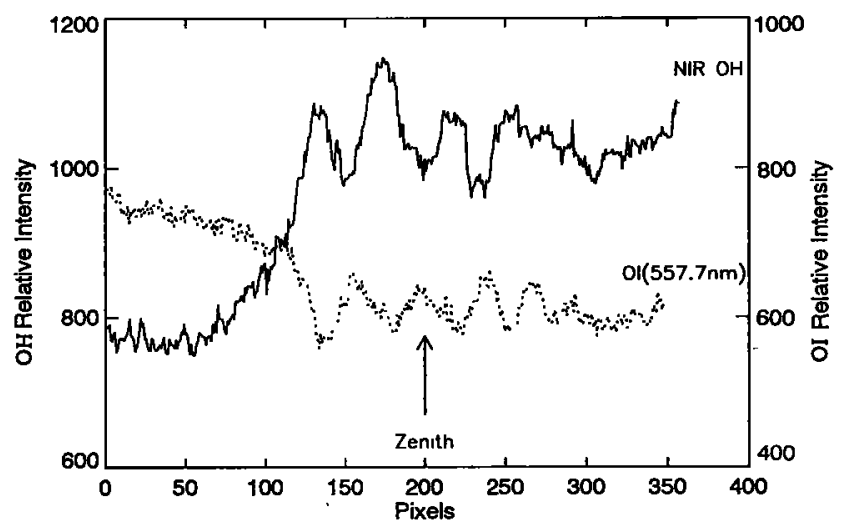

Figure 2. Relative intensity scan across the camera field normal to the wave crests for the $\mathrm{OH}$ and OI data at $\sim 10: 57$ UT when the front passed overhead. Both images have been flat-fielded to remove contributions due to line of sight integration through the nightglow layers and lens vignetting. The OI scan has been shifted horizontally by 8 pixels to compensate for the mean time interval $(\sim 1 \mathrm{~min})$ between the two images. Note the alignment of the peaks in $\mathrm{OH}$ with troughs in $\mathrm{OI}$ and vice versa. depicts this effect well but the magnitude of the contrast is somewhat exaggerated due to the limited dynamic range of the photographic reproduction. Intensity measurements across the camera field in a direction normal to wave crests (Figure 2) quantify the impact of the front on these two emissions. A significant increase in the broad band $\mathrm{OH}$ signal $(\sim 33 \%)$, in the wake of the front, and a corresponding decrease $(-24 \%)$ in OI signal intensity was found. In both cases the amplitudes of the coherent waves trailing the front were considerably less $(\sim 8-$

Table 1. Horizontal wave parameters derived from the image data for each of the four nightglow emissions.

\begin{tabular}{ccccc}
\hline Emission & $\begin{array}{c}\text { Assumed } \\
\text { Height }(\mathbf{k m})\end{array}$ & $\lambda_{\mathbf{h}}(\mathbf{k m})$ & $\mathbf{v}_{\mathbf{h}}\left(\mathrm{ms}^{-1}\right)$ & $\mathrm{T}_{\text {obs }}(\mathrm{min})$ \\
\hline $\mathrm{OH}$ & 87 & $19.3 \pm 0.7$ & $\mathbf{7 5} \pm 2$ & $4.3 \pm 0.2$ \\
$\mathrm{Na}$ & 90 & $18.8 \pm 0.7$ & $\mathbf{7 7} \pm 2$ & $4.1 \pm 0.2$ \\
$\mathrm{O}_{2}$ & 94 & $18.8 \pm 0.9$ & $76 \pm 3$ & $4.1 \pm 0.3$ \\
OI & 96 & $20.1 \pm 0.7$ & $\mathbf{7 7} \pm 2$ & $4.4 \pm 0.2$ \\
Average & & $19.3 \pm 0.7$ & $76 \pm 2$ & $4.2 \pm 0.2$ \\
\hline
\end{tabular}


16\%) than that of the front. A similar, but less contrasted effect was also observed in the $\mathrm{Na}$ and $\mathrm{O}_{2}$ emissions with the $\mathrm{Na}$ images mimicking the $\mathrm{OH}$ data and the $\mathrm{O}_{2}$ images the $\mathrm{OI}$ data. The disturbance was observed for over 3 hours as it progressed from the horizon to horizon and showed no signs of dissipating.

\section{Results and Discussion}

Table 1 lists the horizontal wave parameters derived from the image data. Within the limits of the measurements the wave motion exhibited the same horizontal wavelength at each emission altitude, $\lambda_{\mathrm{h}}=19.3 \mathrm{~km}$, and the same apparent phase speed, $v_{h}=76 \mathrm{~ms}^{-1}$, giving an average observed period, $T_{0 b s}$ of only $4.2 \mathrm{~min}$. Simultaneous measurements using the MF radar located on Kauai (range $\sim 375 \mathrm{~km}$ ) indicate that when this event passed over the Hawaiian Islands (09:30-11:30 UT) the average hourly winds in the direction of the wave motion (azimuth $\sim 126^{\circ} \mathrm{N}$ ) were relatively small throughout the $80-100 \mathrm{~km}$ region at $\sim 17 \mathrm{~ms}^{-1}$ at $\mathrm{OH}$ heights and $<10 \mathrm{~ms}^{-1}$ in the higher altitude emissions (J.R. Isler, private communication). The intrinsic period of the wave motion (i.e. its period measured in a frame of reference moving with the wind) was therefore between 4.6 and 5.5 min. This is close to the local Brunt-Văisälä period, estimated at $\sim 4.8 \mathrm{~min}$ using a mean $\mathrm{OH}$ temperature of $205 \mathrm{~K}$ from the interferometer data and raises several questions concerning the nature and origin of this disturbance.
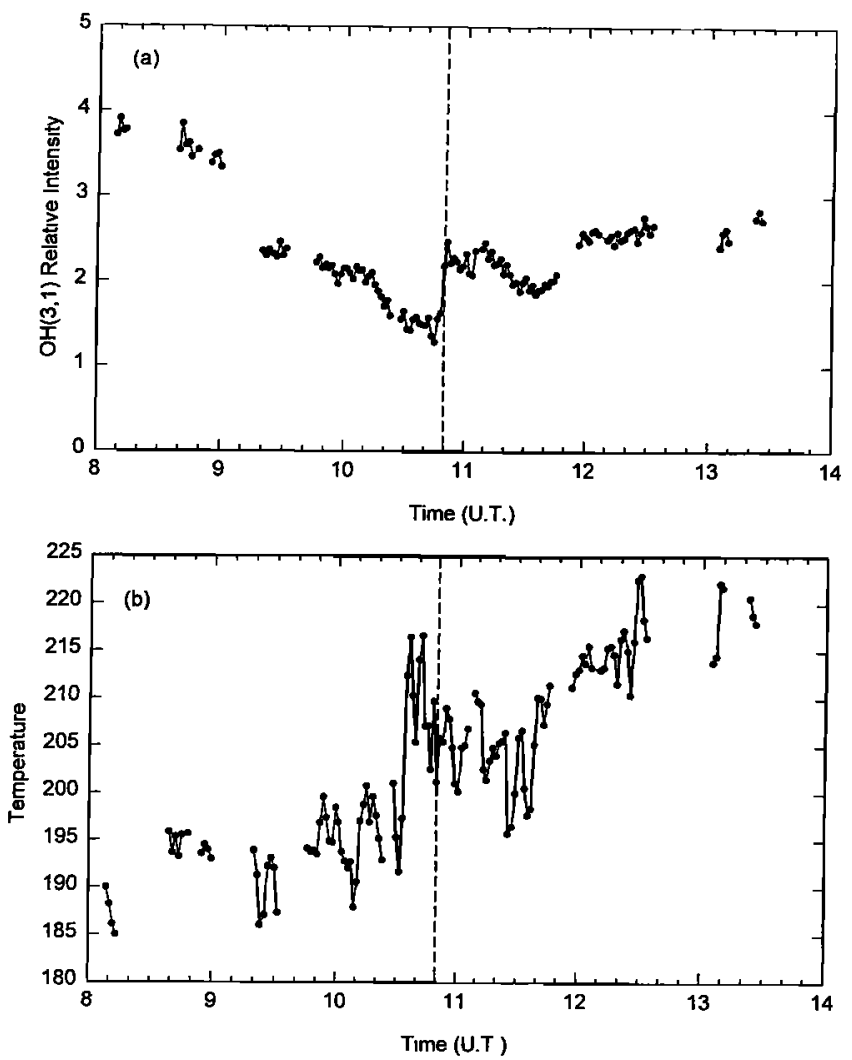

Figure 3. Plots of (a) relative intensity and (b) rotational temperature of the $\mathrm{OH}(3,1)$ band. The data have been averaged and are plotted at $1.5 \mathrm{~min}$ resolution (the temperature data have been further three point smoothed due to noise). Note the sharp transition induced by the passage of the front and the large $(-15$ min) time lag between temperature and intensity.
Extensive wave patterns with apparent periods $<20$ min were observed on many occasions during this campaign. However, only on one previous occasion (during the ALOHA-90 campaign) has such a short apparent wave period been reported [Taylor and Edwards, 1991]. On that occasion (25 March 1990) the display was observed to last for several hours and consisted of a succession of many faint wave crests. The data reported here are quite dissimilar in appearance, consisting of a well defined front and a limited number of wave crests (6-10) progressing behind it. This wave motion stands out in our image data as exceptional in its morphology, clarity and speed of propagation all of which add up to make it a spectacular event. The interferometer data recorded during the transit of this event also point to it being most unusual but for different reasons. Figure 3a shows the intensity of the $\mathrm{OH}(3,1)$ band as measured by the interferometer on this night. The data have been averaged and are plotted at 1.5 min resolution. Prior to the event (08:00-10:50 UT) the $\mathrm{OH}$ intensity dropped steadily by over a factor of two. A decrease of this magnitude during the pre-midnight hours was usual for this campaign, though it normally took place over a five hour period [Lowe and Turnbull, 1995]. However, at 10:50 UT a sudden jump in intensity was detected commensurate with the passage of the front overhead, as determined from the image data. Figure 4 shows an expanded plot of the interferometer data at 23s temporal resolution (with three point smoothing) for this interval. In less than four minutes the $\mathrm{OH}$ intensity increased by over $50 \%$. After this the emission was characterized by several small amplitude variations (12-18\%) associated with the passage of the waves. The average period of this oscillation was found to be $\sim 4.4 \mathrm{~min}$ in good agreement with the image data. Although an intensity change of $50 \%$ is not by itself exceptional the abruptness of the transition (occurring within one cycle of the wave motion) is unprecedented in our data sets. This jump appears more like a discontinuity and is a clear feature of the front-like nature of the disturbance.

The $\mathrm{OH}$ rotational temperature data derived from these measurements is shown in Figure $3 \mathrm{~b}$. The normal nightly temperature variation during the campaign was an increase from a minimum value of around $195 \mathrm{~K}$ at $\sim 06: 00 \mathrm{UT}$ to about $208 \mathrm{~K}$ by 15:00 UT. Prior to the passage of the front the temperature was relatively steady around $195 \mathrm{~K}$. However, at 10:31 UT an abrupt increase of $-20 \mathrm{~K}$ ( $25 \mathrm{~K}$ peak to trough) occurred within a short time interval ( $<4 \mathrm{~min}$ ) in clear association with the transit of the front. Remarkably this transition in temperature preceded the intensity jump by almost 15 minutes suggesting that the thermal disturbance associated with this event was over three cycles ahead of the intensity disturbance as determined from both the interferometer and image data. This result was confirmed by simultaneous temperature measurements using the $\mathrm{OH}(4,2)$ band (data not shown) which are essentially independent from the $(3,1)$ band measurements. Numerous theoretical studies indicate that long period (several hour) gravity waves should exhibit sizable phase shifts between the induced temperature and intensity waves. However, the signature of the front was clearly not wave-like in character raising further important questions concerning the nature of this disturbance and its interaction with the atmosphere.

It is possible that this event may represent a "step-like" disturbance propagating through the $\mathbf{8 0 - 1 0 0 ~} \mathrm{km}$ level rather than a discrete wave packet, as there was no obvious phase shift in the geographic position of the leading edge as detected in the 


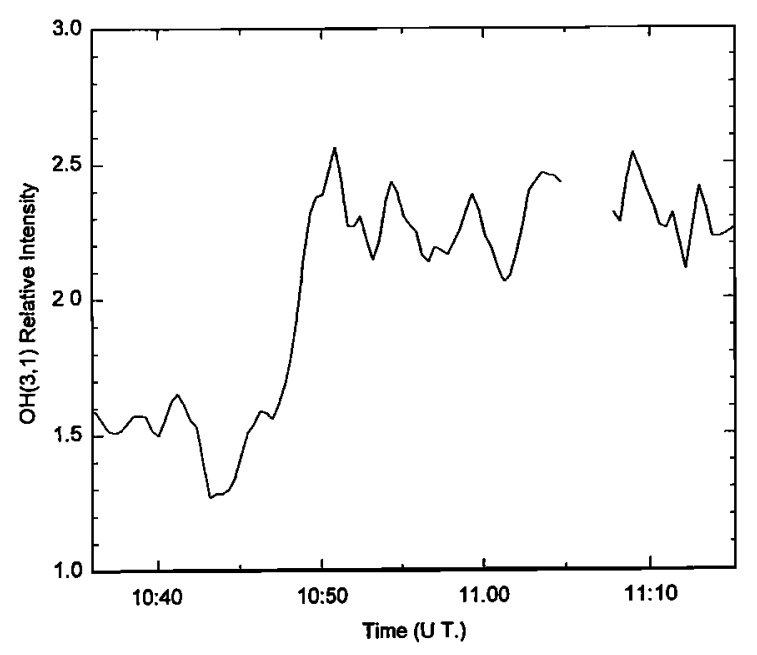

Figure 4. Plot of the $\mathrm{OH}(3,1)$ band intensity data at $23 \mathrm{~s}$ resolution (with three point smoothing) showing the rapid increase in intensity ( $>50 \%$ in less than $4 \mathrm{~min}$ ) due to the front and the ensuing oscillation induced by the waves.

$\mathrm{OH}$ and OI layers (Figure 2). If this were the case then the waves may have been created in-situ in the wake of the passage of a "bore-like" disturbance. Indeed the similarity in morphology of the airglow images to that of a tidal river bore is striking (E.M. Dewan, private communication). Bores and solitons have been observed in the lower atmosphere [Ramamurthy et al., 1990] but not previously in the mesosphere. It is most likely that the waves behind the front are fully ducted waves trapped in the vicinity of the mesopause. The short wave period, close to the Brunt-Väisälä period, and long duration of this event are most consistent with this hypothesis. Freely propagating short period waves of tropospheric origin, on the other hand, would encounter considerable difficulty achieving mesospheric heights.

Another characteristic of this event is the apparent reversal in contrast of the structures as seen in the $\mathrm{OH}$ and $\mathrm{OI}$ data (Figure 2). As sequential images of these emissions are rare it is not known whether this effect is a common occurrence. However, a similar effect was observed on several other occasions during this campaign but they were far less conspicuous. Recent model calculations by Picard et al. [1995] indicate that the observed contrast reversal and the measured temperature change could be explained by a bore-like intrusion simultaneously raising the $O$ layer by $\sim 2 \mathrm{~km}$ and pushing the $\mathrm{OH}$ layer down by the same amount. This would also account for the abrupt nature of the intensity change (within an interval of $<4 \mathrm{~min}$ ). As no such rapid change has been reported previously, it remains to be seen whether the observed phenomena can be best accounted for in terms of a ducted bore-like motion.

\section{Summary}

Observations of gravity wave events of this starkness and clarity are rare. The "front-like" nature of the disturbance was very distinctive and most unusual. These data offer a unique opportunity to discover important information on the origin and manner of propagation of this new type of disturbance and to investigate its impact on the nightglow emission chemistry. Unfortunately on this night, optical data are available only from the UWO and USU instruments co-located on Haleakala. Nevertheless, these joint observations have revealed the following remarkable properties:

a) unusual "front-like" morphology,

b) sudden large "step" in intensity induced by the front,

c) associated sharp jump in $\mathrm{OH}$ rotational temperature,

d) marked time delay between temperature and intensity fronts,

e) companion waves of very short period ( $<5 \mathrm{~min}$ ),

f) high apparent phase speed,

g) coherent progression from horizon to horizon with no sign of dissipation,

h) same disturbance seen in all four emissions (80-100 km), and

i) contrast reversal in the $\mathrm{OH}$ and $\mathrm{OI}(557.7 \mathrm{~nm})$ wave structure.

In total over 100 images of this remarkable event were recorded from which a detailed description of its vertical and horizontal form will be built in due course.

Acknowledgments. We are grateful to J.A. Albetski, ThermoTrex Corp., for arranging our use of the DOE Facility on Haleakala. We thank V. Taylor and Z. Krzeminski for their help with the field measurements and the data analysis. Funding for the image measurements was provided by National Science Foundation (NSF) CEDAR program grant No. ATM-9302844. Partial support for the interferometer measurements and the joint data analysis was provided by the Geophysics Directorate, Air Force Phillips Laboratory, contract No. F19628-93-C-0165 as part of the SOAR program.

\section{References}

Armstrong, E.B., The association of visible airglow features with a gravity wave, J. Atmos, Terr. Phys., 44, 325-336, 1982.

Lowe, R.P., D.N. Turnbull, Comparison of ALOHA-93, ALOHA-90 and ANLC-93 hydroxyl intensity and temperature behavior, Geophys. Res. Lett., this issue, 1995.

Lowe, R.P., K.L. Gilbert, and D.N. Turnbull, High latitude summer observations of the hydroxyl airglow, Planet. Space Sci., 39, 1263$1270,1991$.

Picard, R.H., U.B. Makhlouf, J.R. Winick, M.J. Taylor, D.N. Turnbull, and R.P. Lowe, Airglow response to gravity waves and frontal events in ALOHA-93 (abstract), XXI General Assembly, Boulder, CO, 2-14 July, 1995.

Ramamurthy, M.K., B.P. Collins, R.M. Rauber, and P.C. Kennedy, Evidence of very-large-amplitude solitary waves in the atmosphere, Nature, 348, 314-317, 1990.

Takahashi, H., P.P. Batista, Y. Sahai, and B.R. Clemesha, Atmospheric wave propagations in the mesopause region observed by the $\mathrm{OH}(8,3)$ band, $\mathrm{NaD}, \mathrm{O}_{2} \mathrm{~A}(8645 \AA)$ band and $015577 \AA$ nightglow emissions, Planet. Space Sci., 33, 381-384, 1985.

Taylor, M.J., M. B. Bishop, and V. Taylor, All-sky measurements of short period waves imaged in the $\mathrm{OI}(557.7 \mathrm{~nm}), \mathrm{Na}(589.2 \mathrm{~nm})$ and near infrared $\mathrm{OH}$ and $\mathrm{O}_{2}(0,1)$ nightglow emissions during the ALOHA-93 campaign, Geophys. Res. Lett., this issue, 1995.

Taylor, M.J., and R. Edwards, Observations of short period mesospheric wave patterns: in situ or tropospheric wave generation?, Geophys. Res. Lett., 18, 1337-1340, 1991.

Taylor, M.J., D.N. Turnbull, and R.P. Lowe, Coincident imaging and spectrometric observations of zenith $\mathrm{OH}$ nightglow structure, Geophys. Res. Lett., 18, 1349-1352, 1991.

Taylor, M.J., M.A. Hapgood, and P. Rothwell, Observations of gravity wave propagation in the $\mathrm{OI}(557.7 \mathrm{~nm}), \mathrm{Na}(589.2 \mathrm{~nm})$ and the near infrared $\mathrm{OH}$ nightglow emission, Planet. Space Sci., 35, 413$427,1987$.

M.J. Taylor, Space Dynamics Laboratory, Utah State University, Logan, UT 84322-4145 (e-mail: Taylor@psi.sci.sdl.usu.edu).

D.N. Turnbull and R.P.Lowe, Institute for Space and Terrestrial Science and Physics Department, The University of Western Ontario, London, Ontario, Canada, N6A 3K7 (e-mail: Turnbull@canlon. physics.uwo.ca and Lowe@canlon.physics.uwo.ca).

(Received December 1, 1994; revised August 15; 1995, accepted August 15, 1995) 